\title{
Effects of Synthetic and Processing Methods on Dispersion Characteristics of Nanoclay in Polypropylene Polymer Matrix Composites
}

\author{
T. P. Mohan, K. Kanny \\ Composites Research Group, Department of Mechanical Engineering, Durban University of Technology, Durban, South Africa. \\ Email: kannyk@dut.ac.za
}

Received February $16^{\text {th }}, 2011$; received March 21 ${ }^{\text {th }}, 2011$; accepted May $6^{\text {th }}, 2011$.

\begin{abstract}
This work presents the effect of synthetic procedures (extrusion and casting) on the dispersion characteristics of nano layered silicate clay particles in the polypropylene $(P P)$ polymer matrix. Three different molecular weights PP samples are taken and filled with nanoclay of $1 \mathrm{wt} \%$ and $3 \mathrm{wt} \%$, and these nanocomposites were synthesized by using an extrusion or casting methods. The X-ray diffraction (XRD) and Transmission Electron Microscopy (TEM) is used to characterize the structure and morphology of nanocomposites. Rheological and mechanical results show that the extruded products are better than that of cast products. The outcome of this work is discussed in this paper.
\end{abstract}

Keywords: Polymer-Clay Nanocomposites, Nanocomposites, Nanoclays, Polypropylene, Mechanical Properties

\section{Introduction}

In modern plastics, reinforcement of any inorganic or organic particles is the most common method to improve the properties of the polymer. In particular, Polymer Clay is an interesting and very promising research area due to cost effective and their ease of availability from natural resources. Smectic clays, in particular, montmorillonite (MMT) type of clays are predominantly used as nano fillers in the Polymer Clay Nanocomposites (PCN). Naturally available MMT clays are hydrophilic and they must be made organophilic (hydrophobic) to have compatible with most host polymers, because most of polymers are hydrophobic. Organic treatment is typically accomplished via organic cations, namely onium or quaternary ammonium based salts in MMT clays. When these organotreated MMT clays are filled in to the host polymer matrix, two types of morphologies will form, namely, intercalated and delaminated/exfoliated nanocomposite structure. The intercalated structure is a well-ordered multilayered structure of silicates, where the polymer chains are just inserted into the interlayer spaces (intergallery region). On the other hand, in the exfoliated/delaminated structure the polymer chains separate individual silicate layers well apart, e.g., 80 $100 \AA$ or more, and no longer close enough to interact with each other. Sometimes in exfoliated structure, nanolayers are randomly dispersed in matrix polymer. The aspect ratio (length/thickness) of individual nanolayer of clay is very high in the order of several 100 to 1000 , and therefore contribute to the improved mechanical properties in polymer system along the loading direction and as well as serves as an impermeable medium when the host polymer is exposed to the gas/moisture medium. The maximum utilization of this aspect ratio is fully exploited by an exfoliated structure of PCN composites rather than an intercalated structure. Hence, achieving an exfoliated structure is a real challenge in this PCN system, even though various polymers are used in recent years [1-11].

Polypropylene (PP)-clay nanocomposites is an area of tremendous interest due to wide application of PP polymer in commodity areas and also recent usage in engineering applications. The addition of nanoclay in PP matrix increases the thermal stability, increases physical properties (dimensional stability), improves flame retardant properties (increased thermal-oxidative stability and reduced Heat Release Rate), and improves mechanical properties and fracture properties and gas barrier properties. PP is a relatively inert thermoplastic polymer with non polar characteristics and moreover does not have any reactive functional group. So there cannot be any reaction in PP with alky 1 ammonium ions of organo MMT 
clays in the intergallery regions of clays. Hence, the nanolayer dispersion in PP matrix can be obtained only through proper shear device with controlled processing parameters. Several studies in literature have focused on addition of compatibilizer in PP and clay nanocomposites, effect of organoclay types, thermal and mechanical properties [12-25].

From the literature work of PP-clay nanocomposites it is found that the report on the influence of molecular weight and synthetic methods of PP polymer is not fully exploited. Hence, in this work a detailed insight is given on how the synthetic methods, processing conditions and their resultant structure and morphology affects various molecular weights of PP polymers that are filled with nanoclay particles. Considering the difficulties in achieving the exfoliation structure, such studies are important in PP-clay based nanocomposites because the intercalation and exfoliation structure takes place only through proper control of processing parameters as well as the synthetic methods. In this work, three different molecular weight of PP sample were chosen and nanoclays were added at 1 $\mathrm{wt} \%$ and $3 \mathrm{wt} \%$ and the materials were developed either by extrusion or casting methods.

\section{Experimental Details}

\subsection{Materials}

Polypropylene pellets (melting point $168^{\circ} \mathrm{C}$ ) were procured from Chempro, South Africa. Three different molecular weights $\left(M_{w}\right)$ of PP polymer $\left(M_{w}\right.$ of $3.5 \times 10^{5}$, $2.06 \times 10^{5}$ and $1 \times 10^{5}$ ) were procured from Chempro, South Africa. $M_{w}$ of $3.5 \times 10^{5}$ is designated at high molecular weight polypropylene (LM-PP), $M_{w}$ of $2.06 \times 10^{5}$ is designated at medium molecular weight polypropylene (MM-PP) and $M_{w}$ of $1 \times 10^{5}$ is designated at low molecular weight polypropylene (LM-PP) in future discussions. Cloisite 15 A nanoclay was obtained from Southern Clay Products, USA. This Cloisite 15 A clay is montmorillonite clay that was organically modified with a quaternary ammonium salt.

\subsection{Nanocomposite Preparation}

The PP-clay nanocomposites was prepared by using two methods, namely, extrusion and casting. In extrusion method, the polypropylene pellets and the nanoclay were combined in a REIFFENHAEUSER single screw extruder. The extruder has a $40 \mathrm{~mm}$ diameter single rotating screw with a length/diameter ratio (L/D) of 24 and driven by a $7.5 \mathrm{~kW}$ motor. Extruder has got three heating zones along the length of the screw as follows: Zone 1 (Hopper or pellet loading end), Zone 2 (centre region of screw) and Zone 3 (extrusion end). The melt mixing conditions were kept at constant temperature of $190^{\circ} \mathrm{C}$ (Zone 1), $230^{\circ} \mathrm{C}$ (Zone 2) and $230^{\circ} \mathrm{C}$ (Zone 3), and the screw speed is kept at $80 \mathrm{rpm}$. In casting method, the PP pellets and nanoclays of desired weight content are mixed together by mechanical mixer at room temperature and then heated to its melt temperature using a LABCON HTR2 environmental heating chamber. After placing in the molten condition for the desired time (15 minutes), the molten sample was poured in the aluminium mould with dimensions of $275 \times 175 \times 10 \mathrm{~mm}$, and allowed it to set at room temperature.

\subsection{Characterization}

The structure of nanocomposites is studied by using XRD and TEM methods. A Philips PW1050 diffractometer was used to obtain the X-Ray diffraction patterns using $\mathrm{CuK} \alpha$ lines $(\lambda=1.5406 \AA)$. The diffractrograms were scanned from $2.5^{\circ}$ to $12^{\circ}(2 \theta)$ in steps of $0.02^{\circ}$ using a scanning rate of $0.5^{\circ} \mathrm{min}$. Microscopic investigation of selected nanocomposite specimens at the various weight compositions were conducted using a Philips CM120 BioTWIN transmission electron microscope with a 20 to $120 \mathrm{kV}$ operating voltage. The specimens were prepared using a LKB/Wallac Type 8801 Ultramicrotome with Ultratome III 8802A Control Unit. Ultra thin transverse sections, approximately $80-100 \mathrm{~nm}$ in thickness were sliced using a diamond coated blade.

Melt Flow Index is measured for test samples as per ASTM D1238 testing method. Thermal Analyzer DSC instrument is carried out for composites series to study the thermal properties. Heating were carried out from room temperature to $200^{\circ} \mathrm{C}$ at the heating rate of $10^{\circ} \mathrm{C} / \mathrm{min}$ and two heating scans have been conducted on the test specimen. In heat -1 scanning, the melting temperatures $\left(T_{m l}\right)$ were observed. In these curves, $\Delta \mathrm{H}(\mathrm{J} / \mathrm{g})$ value of melting peak is measured and the $\%$ crystallinity is measured by comparing with the theoretical $100 \%$ crystalline PP melting peak value $(115 \mathrm{~J} / \mathrm{g})$ [26]. The \% crystallinity is calculated by taking the ratio of $\Delta \mathrm{H}$ of melting of test sample to the $\Delta \mathrm{H}$ of $100 \%$ crystalline PP polymer. Once the polymer is taken to molten state, it is rapidly cooled to room temperature using liquid nitrogen and again heat -2 scan is conducted up to $200^{\circ} \mathrm{C}$. In heat -2 scan, crystallization temperature $\left(T_{c h}\right)$ and actual melting point $\left(T_{m}\right)$ of material is measured. In a special case, once the polymer is taken to molten state (heat -1) it is slowly cooled at the rate of $10^{\circ} \mathrm{C} / \mathrm{min}$ to measure the crystallization temperature on cooling $\left(T_{c c}\right)$. Crystallization rate is measured in composites series by keeping the samples at their respective crystallization temperature $\left(T_{c h}\right)$ at different minutes followed by rapid quenching using cold water to room temperature at the rate of 
$100^{\circ} \mathrm{C} / \mathrm{min}$. The quenched specimen is further heat scanned using DSC to measure the \% crystallinity using above mentioned formula.

\subsection{Mechanical Testing}

Tensile tests were performed on virgin PP and the nanocomposite specimens using the LLOYDS Tensile Tester fitted with a $20 \mathrm{kN}$ load cell. The tensile tests were performed at a crosshead speed of $1 \mathrm{~mm} / \mathrm{min}$ in accordance with the ASTM D3039 standard. Five tensile specimens is taken and the average value is considered for plotting stress-strain curves. It is envisaged that the standard deviation of all the test specimen values are within 3\%. The fracture surfaces of tensile specimens were examined by using JEOL JSM 840A scanning electron microscope (SEM).

\section{Results and Discussions}

\subsection{Structure and Properties}

Table 1 shows the melt flow behaviour of PP-clay series. The result shows that the melt viscosity of all the PP polymer series (LM, MM and HM) is almost constant. The addition of clay increases the melt viscosity of the polymer. The rate of increase in viscosity is higher for HM-PP-clay series than other series. The melting point of PP series is shown in Figure 1 of DSC heat 1 scan. It shows that the melting point of PP polymer series are
Table 1. Melt flow index of PP-clay series.

\begin{tabular}{cc}
\hline Material & MFI, $\mathbf{g} / \mathbf{1 0}$ min \\
\hline LM-PP & 11 \\
LM-PP $+1 \%$ clay & 10 \\
LM-PP $+3 \%$ clay & 9 \\
MM-PP & 11 \\
MM-PP $+1 \%$ clay & 9 \\
MM-PP $+3 \%$ clay & 8 \\
HM-PP & 11 \\
HM-PP $+1 \%$ clay & 7 \\
HM-PP $+3 \%$ clay & 6 \\
\hline
\end{tabular}

almost constant. However, in heat 2 scanning of DSC (Figures 2 and 3) result shows that the crystallization temperature ( $T_{c h}$ and $\left.T_{c c}\right)$ of LM-PP is higher than that of other PP series. The effect of nanoclay on the DSC properties is shown in Table 2. Nanoclay addition increases the $T_{c h}$ and $T_{c c}$ temperature of PP series. The rate of increase of crystallization temperature is lesser for HM-PP series than other PP series. The melting point of PP is by and large unaffected due to the presence of nanoclay. The increased crystallization behaviour of PP-clay nanocomposites is examined by studying the rate of crystallization formation. Table 3 shows the $\%$ crystallization achieved at various time intervals. It is observed that the rate of crystallization formation is higher in nanoclay filled PP composites. Among the nanocomposite series, LM-PP series filled with nanoclay shows higher rate of crystallization formation. As LM-PP

Table 2. DSC heating result of nanocomposites.

\begin{tabular}{cccccc}
\hline Material & $\boldsymbol{T}_{\boldsymbol{m} \mathbf{1}}$ & \% crystallinity & $\boldsymbol{T}_{\boldsymbol{c h}}$ & $\boldsymbol{T}_{\boldsymbol{m}}$ & $\boldsymbol{T}_{\boldsymbol{c c}}$ \\
\hline LM-PP & 168 & 62 & 114 & 168 & 119 \\
LM-PP + 1\% clay & 169 & 71 & 117 & 169 & 125 \\
LM-PP + 3\% clay & 169 & 74 & 116 & 169 & 127 \\
MM-PP & 169 & 56 & 112 & 166 & 117 \\
MM-PP + 1\% clay & 170 & 62 & 115 & 168 & 123 \\
MM-PP + 3\% clay & 170 & 64 & 113 & 167 & 125 \\
HM-PP & 169 & 54 & 112 & 166 & 117 \\
HM-PP + 1\% clay & 170 & 59 & 115 & 167 & 121 \\
HM-PP + 3\% clay & 169 & 61 & 114 & 167 & 122 \\
\hline
\end{tabular}

Table 3. Crystallization behaviour PP-clay series.

\begin{tabular}{|c|c|c|c|c|c|c|c|c|c|}
\hline \multirow{2}{*}{ Time, min } & \multicolumn{3}{|c|}{ LM-PP series } & \multicolumn{3}{|c|}{ MM-PP series } & \multicolumn{3}{|c|}{ HM-PP series } \\
\hline & $0 \%$ clay & $1 \%$ clay & $3 \%$ clay & $\mathbf{0} \%$ clay & $1 \%$ clay & $3 \%$ clay & 0\% clay & $1 \%$ clay & $\begin{array}{l}3 \% \\
\text { clay }\end{array}$ \\
\hline 0 & 0 & 0 & 0 & 0 & 0 & 0 & 0 & 0 & 0 \\
\hline 1 & 17 & 24 & 26 & 13 & 16 & 17 & 12 & 14 & 16 \\
\hline 2 & 36 & 41 & 43 & 30 & 36 & 38 & 28 & 32 & 34 \\
\hline 3 & 47 & 61 & 66 & 41 & 46 & 47 & 38 & 43 & 45 \\
\hline 5 & 62 & 71 & 74 & 56 & 62 & 64 & 54 & 59 & 61 \\
\hline
\end{tabular}




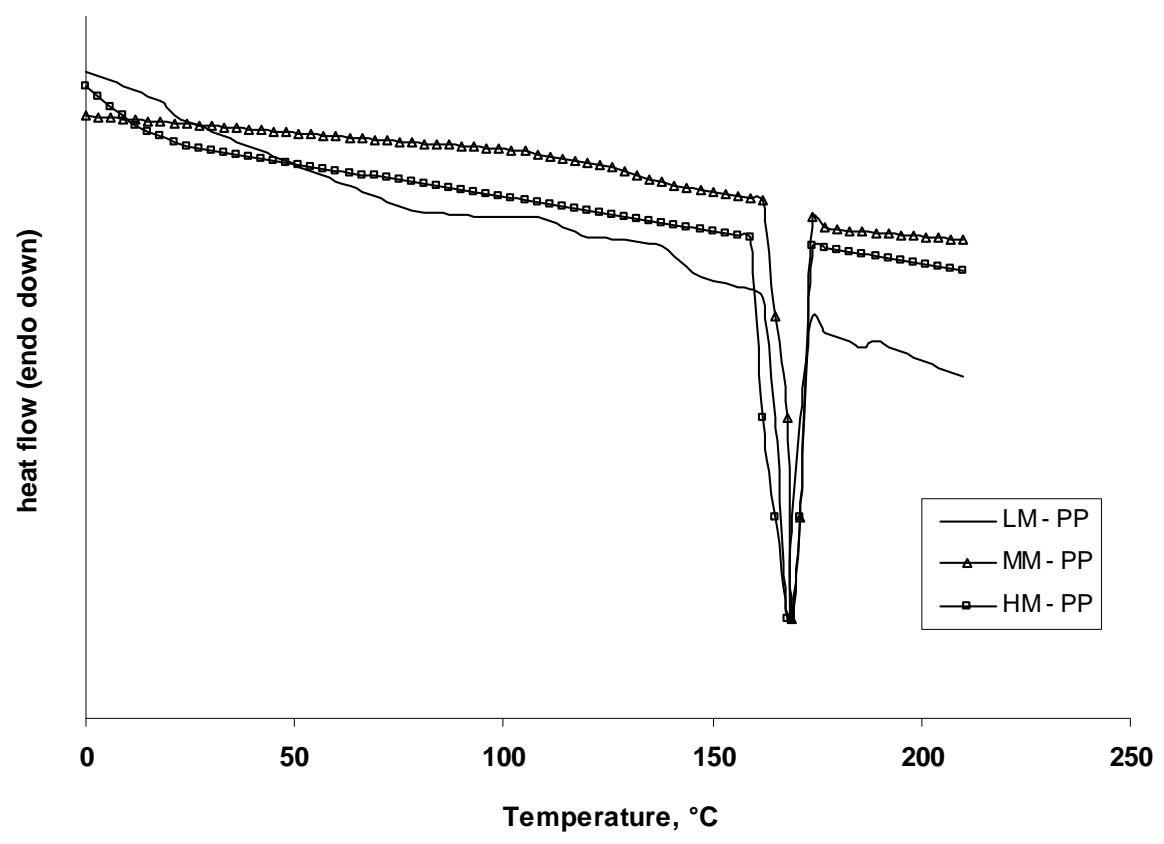

Figure 1. DSC heat -1 scan of PP series.

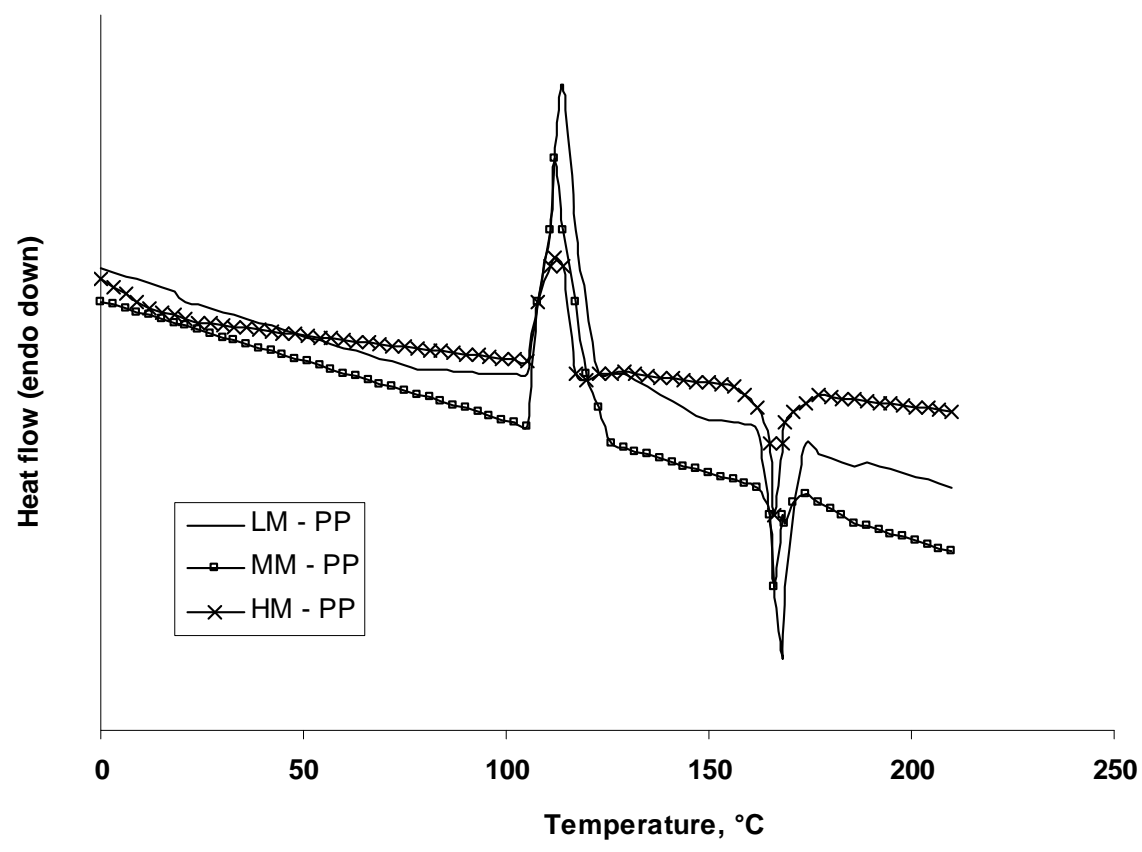

Figure 2. DSC heat -2 scan of PP series.

haslower molecular weight, the crystal formation is relatively easier than that of higher molecular weight PP nanoclay composites.

The increased crystallization rate and $\%$ crystallinity of nanoclay filled PP composites suggests that the nanoclay acts as a nucleating agent. The nucleating behaviour is further examined by studying the Avarami kinetic equation. Equation 1 shows the general Avarami equation as a function of relative crystallinity $\left(X_{f}\right)$ and time to achieve $X_{f}$.

$$
X_{r}(t)=1-e\left(-K t^{n}\right)
$$

where $K$ and $n$ are constants that are considered as the important parameters for crystallization mechanisms. 


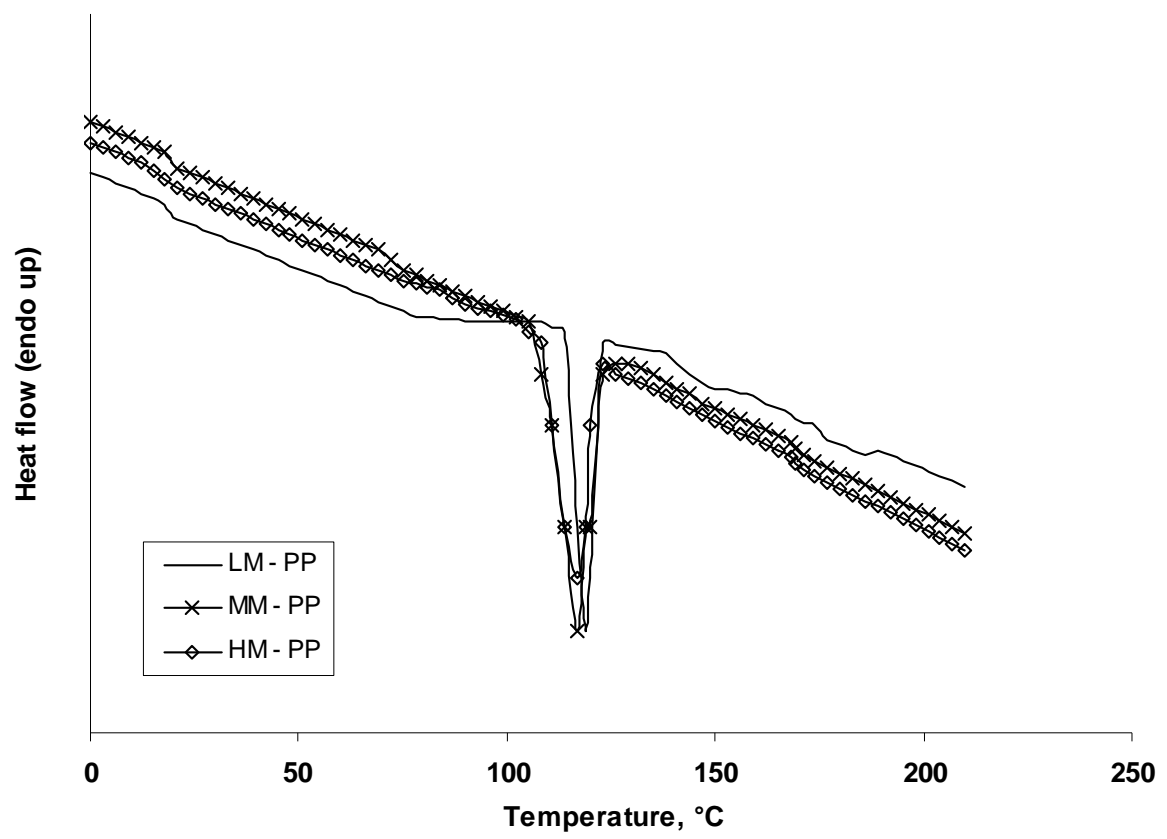

Figure 3. DSC cooling curves of PP series.

The values of $K$ and $n$ can be calculated by plotting $\log \left[-\ln \left(1-X_{r}\right)\right]$ vs. $\log (t) . n$ and $\log (K)$ values are the slope and intercept values respectively of the Avarami's plot. The Avarami plot for PP series and LM-PP-nanoclayseries is showed in Figures $\mathbf{4}$ and $\mathbf{5}$ respectively. The Avarami's kinetic constants, namely, $n$ and $K$ values are shown in Table 4. The remarkable increase in kinetic constant $(K)$ and crystallization rate; decreased $n$ value suggests that the nanoclay acts as the nucleating agent in the system and hence increase the \% crystallinity and rate.

Further, this rheological effect of nanoclay on structure and morphology is planned to examine for PP polymer. Hence, the structure and morphology of extruded samples is examined by using XRD and TEM methods. Figure 6 shows the XRD patterns of PP-clay series. Nanoclay (Cloisite 15A) shows the diffraction peak of $2 \theta$ at $3.3^{\circ}$ and corresponds to interlayer spacing of nanoclay ( $d$-spacing) of $26.75 \AA$ (calculated from Bragg's diffraction law of $2 d \operatorname{Sin} \theta=\mathrm{n} \lambda$ ). HM-PP $+3 \mathrm{wt} \%$ clay shows the diffraction peak at of $2 \theta$ at $3.10^{\circ}$ and corresponds to interlayer spacing of nanoclay ( $d$-spacing) of $28.47 \AA$. This suggests that the interlayer spacing of nanoclay is increased by about $1.72 \AA$ due to the presence of matrix polymer in the interlayer region of nanoclay. The presence of matrix polymer in the interlayer regions increases the interlayer spacing of the nanoclays and more over the results further shows that the nanolayers are arranged parallel to each other which is a typical intercalated structure. In MM-PP - $3 \mathrm{wt} \%$ nanoclay, the diffraction
Table 4. Avarami's kinetic constants of PP-clay series.

\begin{tabular}{ccc}
\hline Material & $\boldsymbol{n}$ & $\boldsymbol{k}$ \\
\hline LM-PP & 1.02 & 0.15 \\
LM-PP + 1\% clay & 0.97 & 0.26 \\
LM-PP + 3\% clay & 0.97 & 0.29 \\
MM-PP & 1.10 & 0.08 \\
MM-PP + 1\% clay & 1.06 & 0.14 \\
MM-PP + 3\% clay & 1.05 & 0.16 \\
HM-PP & 1.12 & 0.06 \\
HM-PP + 1\% clay & 1.10 & 0.10 \\
HM-PP + 3\% clay & 1.05 & 0.13 \\
\hline
\end{tabular}

peak occurs at $2 \theta$ of $2.98^{\circ}$ and this corresponds to the interlayer spacing of clay of $29.62 \AA$. The result shows that in MM-PP-3 wt\% clay composites, the structure is an intercalated structure with increased interlayer spacing of nanolayers than that of HM-PP $-3 \mathrm{wt} \%$ clay composites. In the case of LM-PP $-3 \mathrm{wt} \%$ nanocomposite, no diffraction peak is observed and this suggests that the nanolayers of clay could have randomly dispersed in the matrix polymer or the clay nanolayers are separated well apart ( $>$ $80 \AA$ ) so that Bragg diffraction cannot occur due to $\mathrm{CuK \alpha}$ lines. This type of structure is called an exfoliated structure or ordered exfoliated structure. The results further shows that the low molecular weight based PP-clay composites form an exfoliated structure and whereas higher molecular weight based PP-clay composites form an intercalated structure. To further understand the dispersion of clay in the polymer matrix, TEM is taken for these composites are taken and shown in Figure 7. Figure $\mathbf{7}$ is the bright field TEM pictures of nanoclay. 


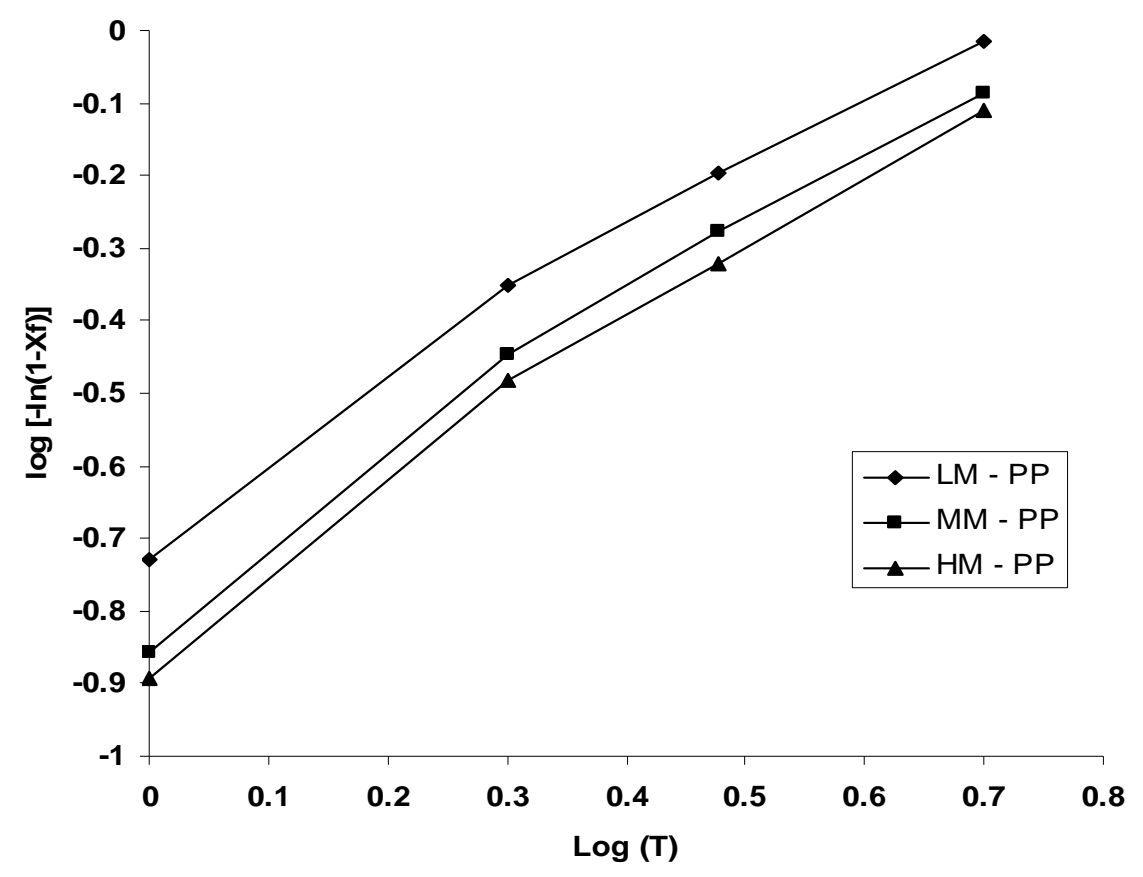

Figure 4. Avarami plot of PP series.

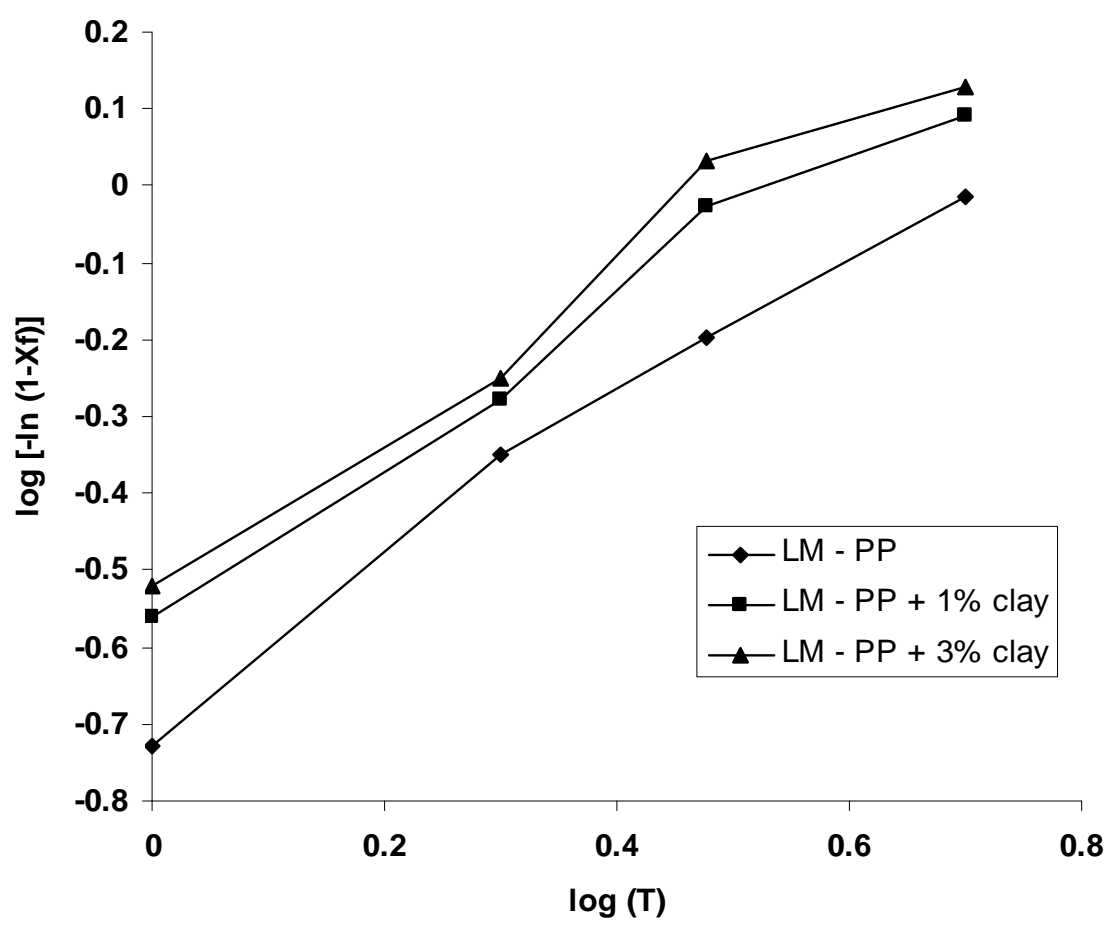

Figure 5. Avarami plot of LM-PP with clay series.

filled PP composites, in which the bright phase in the TEM picture is the matrix phase and the dark phase is the particle phase. LM-PP $+3 \mathrm{wt} \%$ nanoclay composites shows the a well separated distribution nanolayers in the matrix and such structure is the exfoliated structure.
MM-PP + $3 w t \%$ clay and HM-PP $+3 w t \%$ clay composites shows the parallel arrangement of nanolayers in the polymer matrix and such structure is called an intercalated structure. Further more, the TEM pictures supports the XRD data of nanocomposite structure. The pos- 


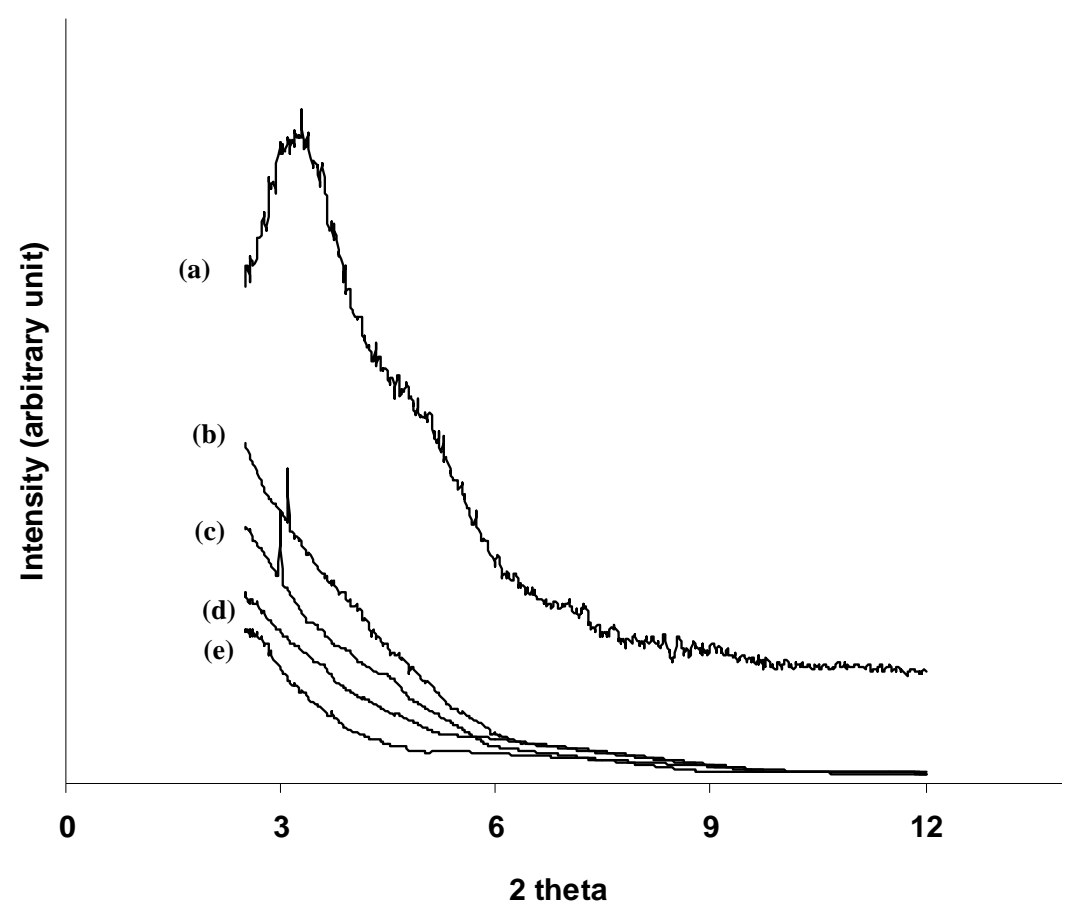

Figure 6. XRD pattern of (a) organoclay, (b) HM-PP + 3 wt \% clay, (c) MM-PP + 3 wt \% clay, (d) MM-PP + 1 wt $\%$ clay and (e) LM-PP + 3 wt \% clay.

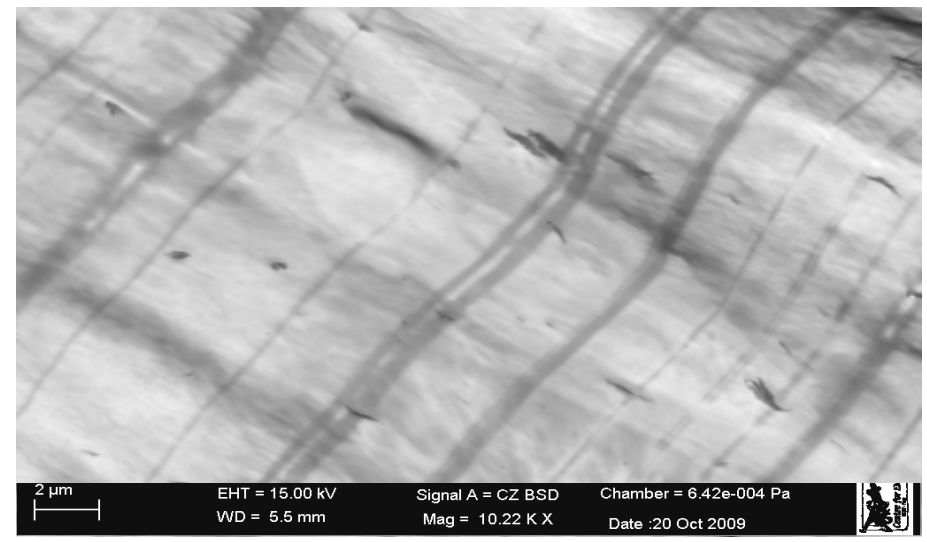

(a)

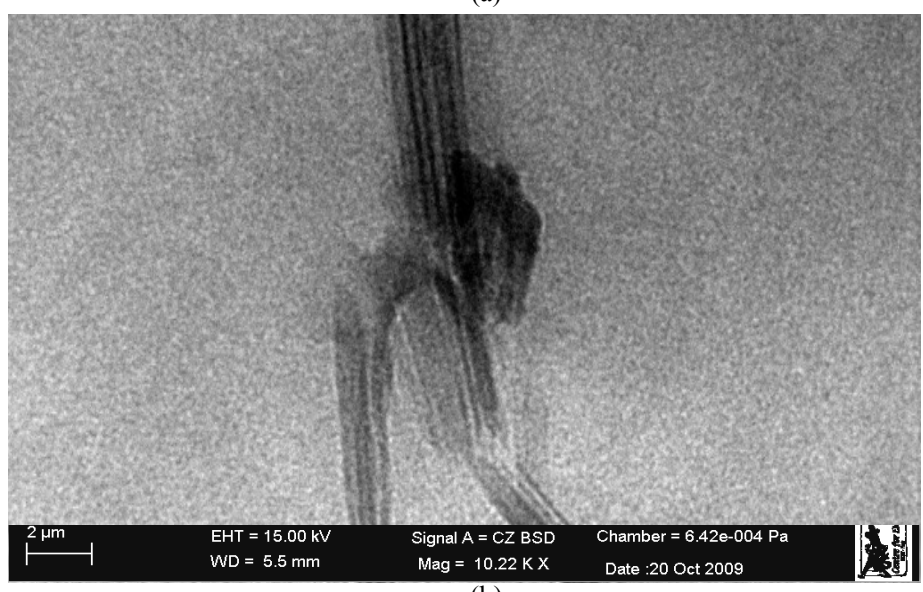

(b) 


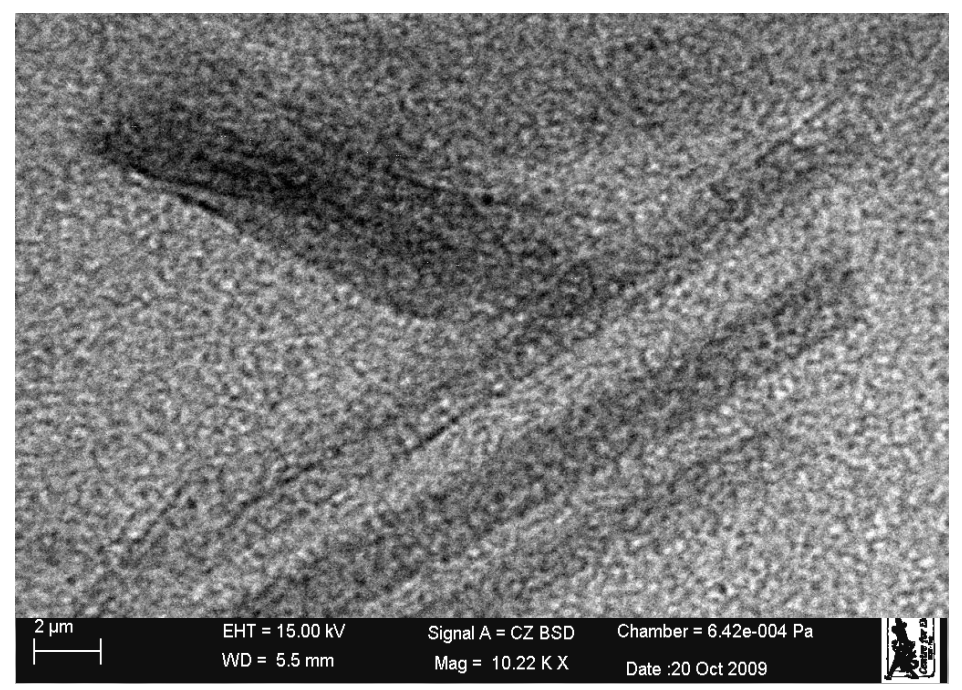

(c)

Figure 7. TEM of (a) LM-PP + 3 wt \% clay, (b) MM-PP + 3 wt \% clay, (c) HM-PP + 3 wt \% clay.

sible reason for intercalated structure in HM-PP and MM-PP series could be due to the high viscosity of polymer and clay mixture that could have induced low shear force during processing. Moreover, all the PP-clay series are processed at same shear force and this force might be lesser to exfoliate the clays in higher molecular weight PP composites.

The effect of this reheological, structure and morphology of nanoclay filled PP composites is further examined by studying the tensile properties of the composites. Figure 8 shows the tensile stress-strain curves of PP series. The curves show that the behaviour of PP series under loading is different from each other. HM-PP shows increased failure strain than that of other PP series, whereas it shows lowest modulus than other PP series. Table 5 and Figure 9 show the effect of nanoclay on tensile properties of various PP series. This result shows that the addition of nanoclay in PP improves the tensile modulus, strength and failure strain in all the PP series. The increased modulus in nanocomposites is due to the molecular level distribution of the clays in the polymer matrix. The nanoclay increases the molecular strength due to the nanolevel distribution of the particles and thereby increases the modulus under loading condition. The possible increase in the strength of the nanocomposites is due to the nanoclay particles acts as a crack stoppers or initiates the crack growth at higher loading level. In addition to these factors, the extended deformation mechanisms could have also caused the composites to fail at higher strength level. To further understand this deformation mechanisms, SEM of the composites specimens were taken and shown in Figure 10. The fracture surface of LM-PP is smooth and the crack has propagated in the material with several branched marks caused due to the propagation of crack front. On the other hand, fracture surface of LM-PP $+3 \mathrm{wt} \%$ clay shows the different fracture morphology. The crack surface shows the extended deformations (shown in circles) that caused the material to fail in higher strain level. Also, the crack surface shows the failure occurs under the cavitation mechanisms (represented by arrows). In this cavitation mechanism, the propagating crack surrounds the particle front and will propagate further at higher loading level until material fails. Hence, these factors have caused the increased tensile properties of nanoclay filled PP polymer composites. On observing Table 5, it further shows that the rate of improvement in tensile properties of high molecular weight PP-clay composite series (MM-PP and HM-PP) is slower than that of other series. The possible cause of this could be the formation of intercalated structure in these nanocomposites. When compared to intercalated structure, exfoliated structure provides better improvement in properties due to the random distribution of nanolayers of clays as well individual dispersion of nanolayers of clays. In the intercalated structure, group of nanolayers forms a stacking sequence and orient in particular direction and this reduces the dispersion ability of individual nanolayers and also reduces the net aspect ratio of the nanolayers (length/thickness). Therefore, better improvement in properties can be obtained in intercalated structure if the nanolayers are dispersed randomly or forms into an exfoliated structure. Hence, to obtain the better dispersion of intercalated structures, the processing parameters were varied and examined. 


\subsection{Effect of Processing Parameters on Structure and Properties of Nanocomposites}

In order to improve the dispersion quality of the nanolayers of clays particles in high molecular weight PP polymer matrix (MM and HM series), it is decided to change the processing parameters. Two parameters were varied in the extrusion processing, namely, increased temperature and increased melt shear force. In the first

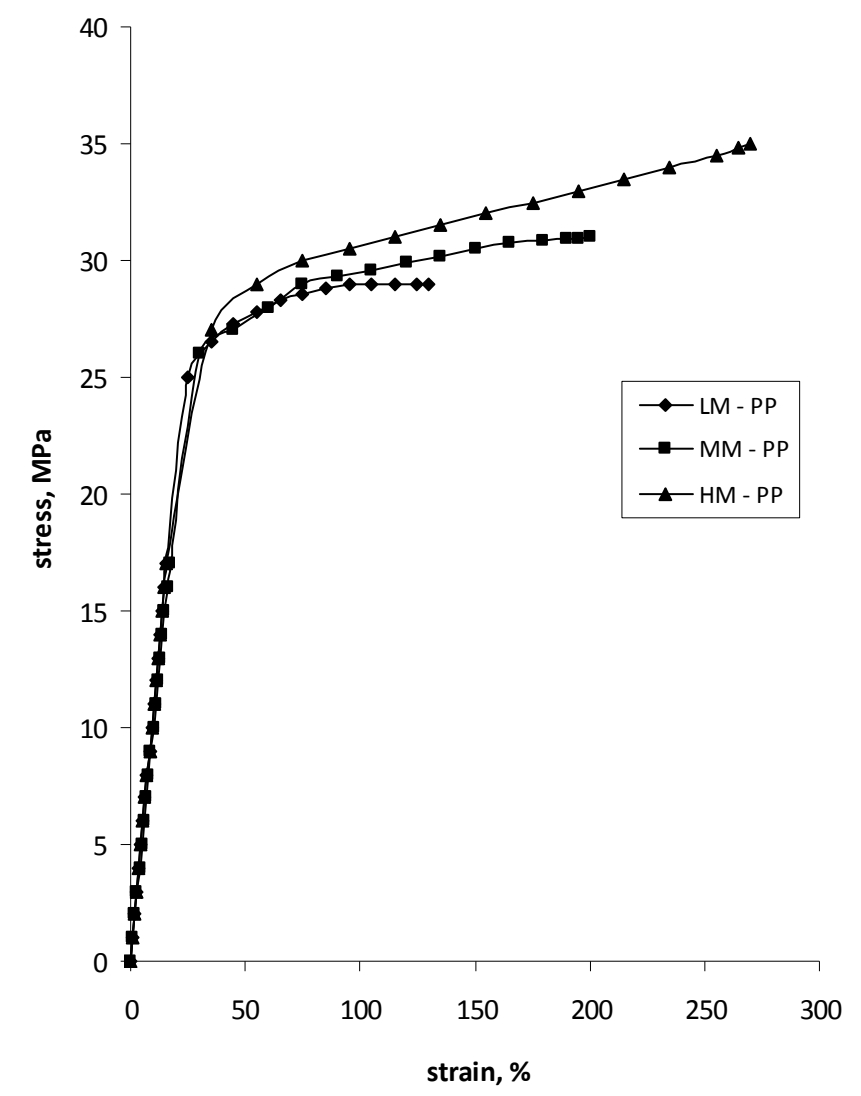

Figure 8. Tensile stress-strain curves of PP series.

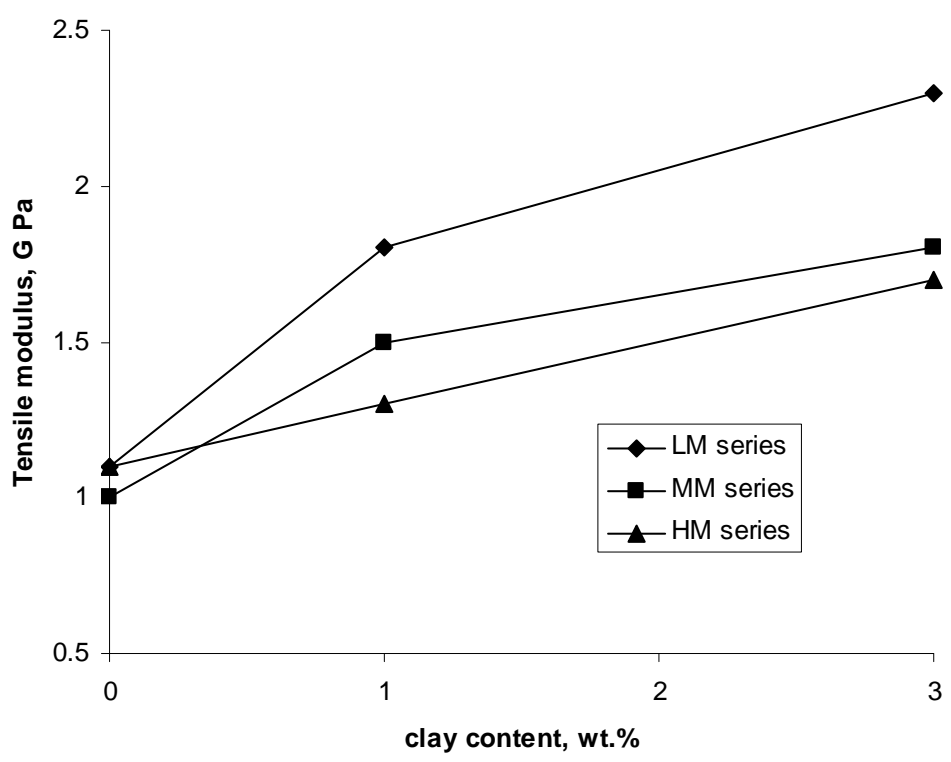

(a) 


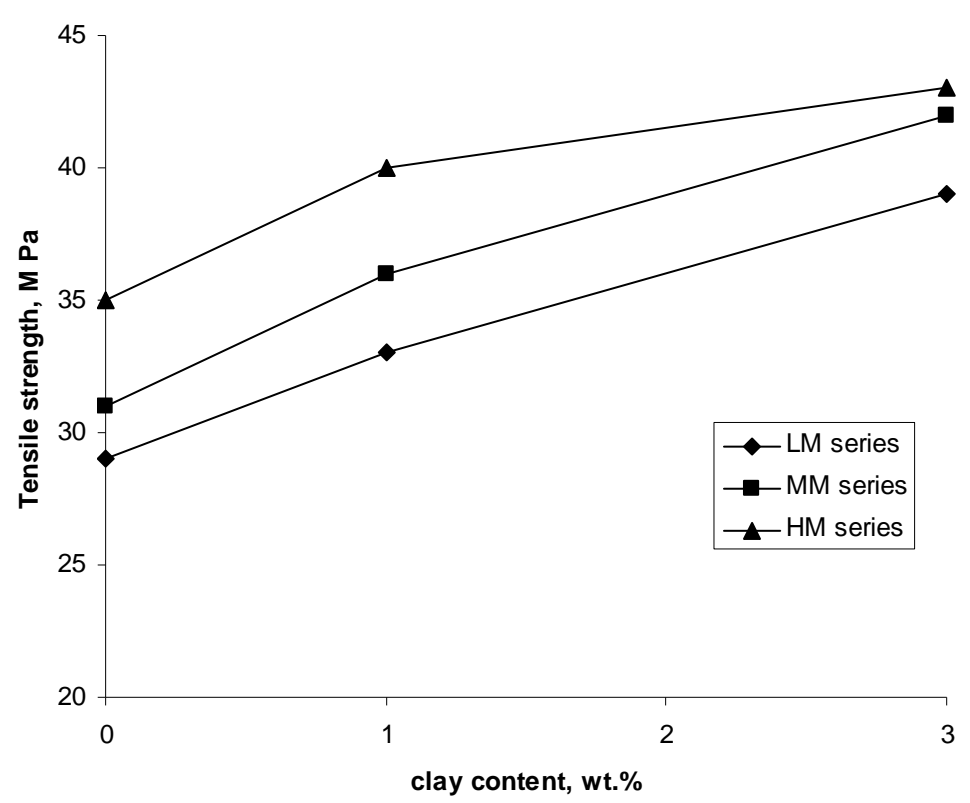

(b)

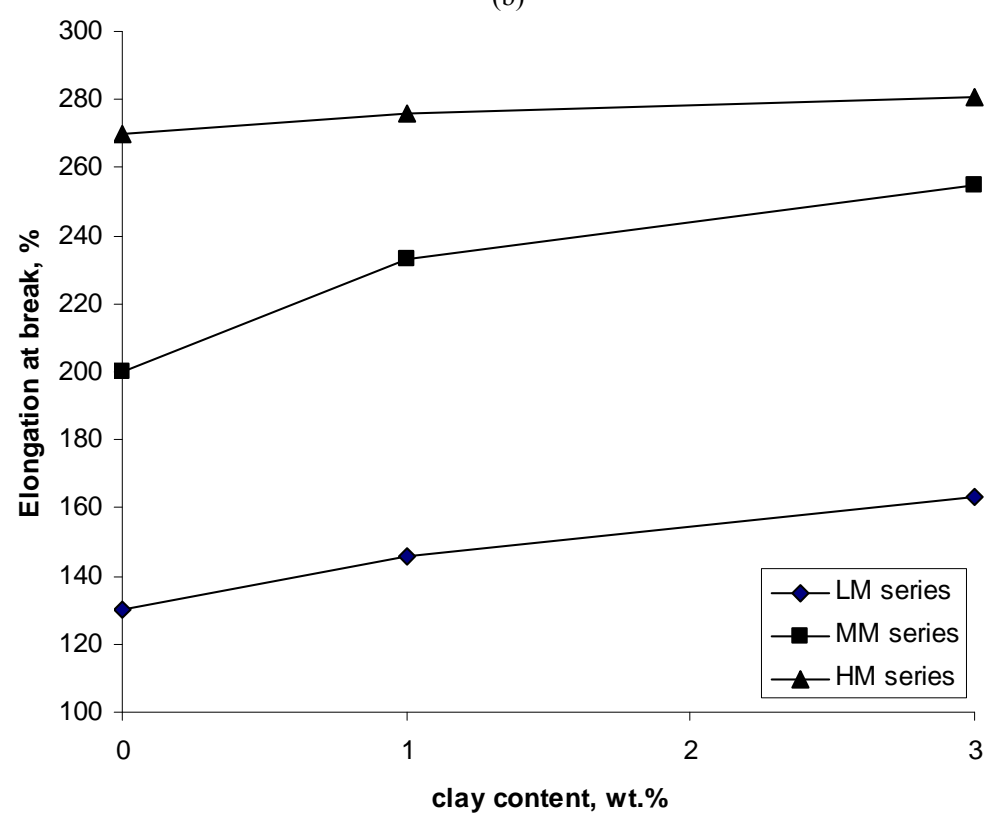

Figure 9. Effect of nanoclay on (a) tensile modulus, (b) tensile strength and (c) elongation at break of PP series.

Table 5. Tensile properties of PP-clay series.

\begin{tabular}{cccc}
\hline \multirow{2}{*}{ Material } & Modulus & Strength & Elongation \\
\cline { 2 - 4 } & GPa & MPa & \% \\
\hline LM-PP & 1.1 & 29 & 130 \\
LM-PP + 1\% clay & 1.8 & 33 & 146 \\
LM-PP + 3\% clay & 2.3 & 36 & 163 \\
MM-PP & 1 & 31 & 200 \\
MM-PP + 1\% clay & 1.35 & 36 & 233 \\
MM-PP + 3\% clay & 1.7 & 38 & 255 \\
HM-PP & 1.1 & 35 & 270 \\
HM-PP + 1\% clay & 1.3 & 43 & 276 \\
HM-PP + 3\% clay & 1.7 & 49 & 281 \\
\hline
\end{tabular}




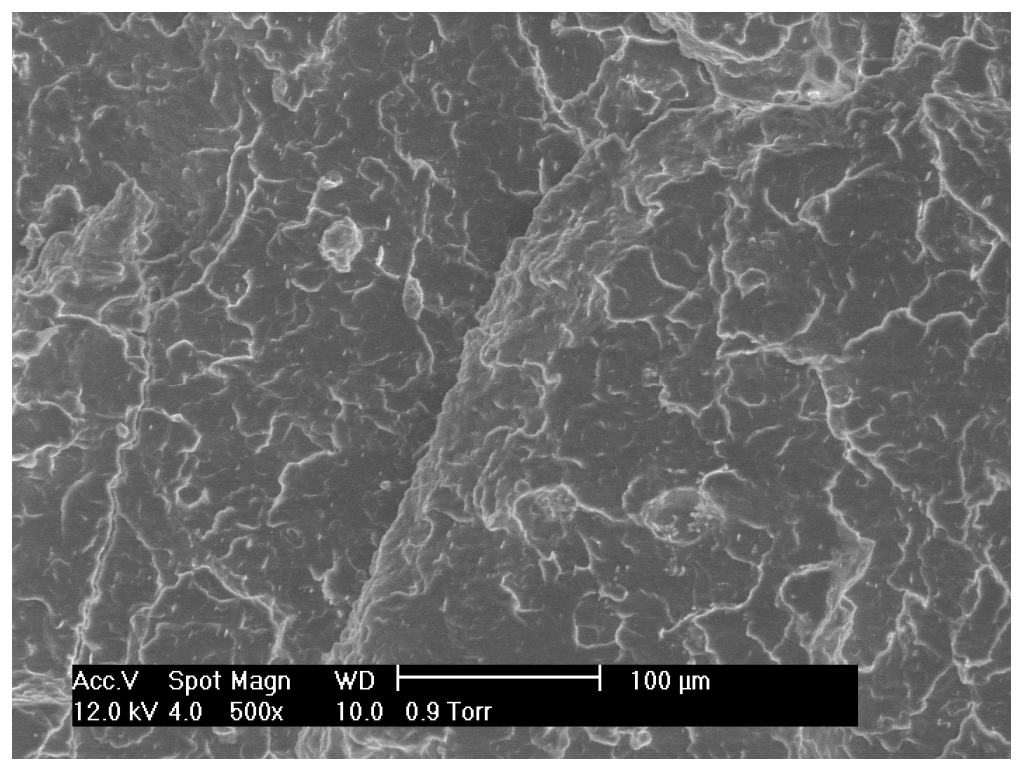

(a)

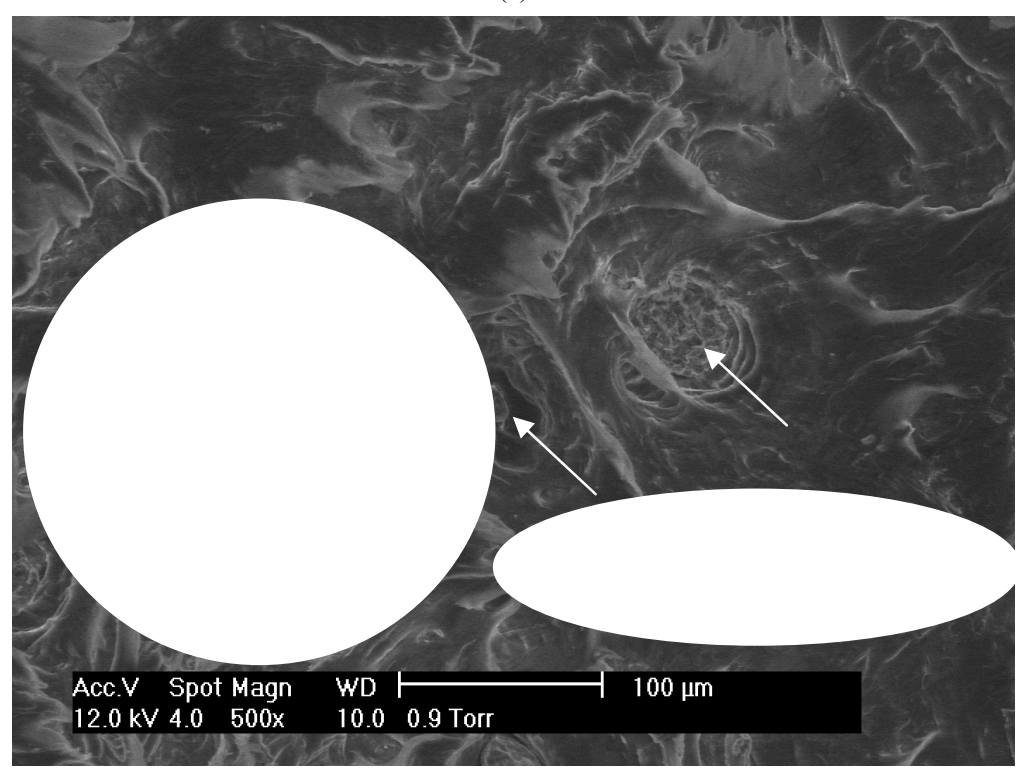

(b)

Figure 10. SEM of fracture surface of (a) LM-PP and (b) LM-PP + 3 wt \% nanoclay.

case, the extrusion processing temperature is varied from $230^{\circ} \mathrm{C}$ to $245^{\circ} \mathrm{C}$ and the resultant change in the XRD pattern over previous method is shown in Figure 11. Figure 11 shows the XRD pattern of MM-PP and HM-PP polymer filled each with $3 \mathrm{wt} \%$ clay. The figure shows a diffraction peak at $2 \theta$ of $2.92^{\circ}$ and $2.82^{\circ}$ and corresponds to the interlayer spacing of $28.47 \AA$ and $29.62 \AA$ for MM-PP $+3 \mathrm{wt} \%$ clay and HM-PP $+3 \mathrm{wt} \%$ clay composites respectively. The results show that the formation of intercalated nanocomposite structure with improved $d$-spacing over the previous processing method conditions $\left(230^{\circ} \mathrm{C}\right)$. In the another case of processing, the shear force is increased by changing screw rpm from 80 $\mathrm{rpm}$ to $120 \mathrm{rpm}$ and keeping the processing temperature constant at $230^{\circ} \mathrm{C}$. Figure 12 shows the XRD pattern of MM-PP and HM-PP polymers filled each with $3 \mathrm{wt} \%$ nanoclay that were processed by high shear force. The XRD pattern shows the absence of any diffraction peaks. This suggests that the structure is an exfoliated structure. To further under stand the dispersion level of nanoclays in the polymer matrix, TEM of these processed samples were taken and shown in Figure 13. Figure 13 is the TEM of HM-PP $+3 \mathrm{wt} \%$ nanoclay processed at two different conditions. It is observed that higher processed 


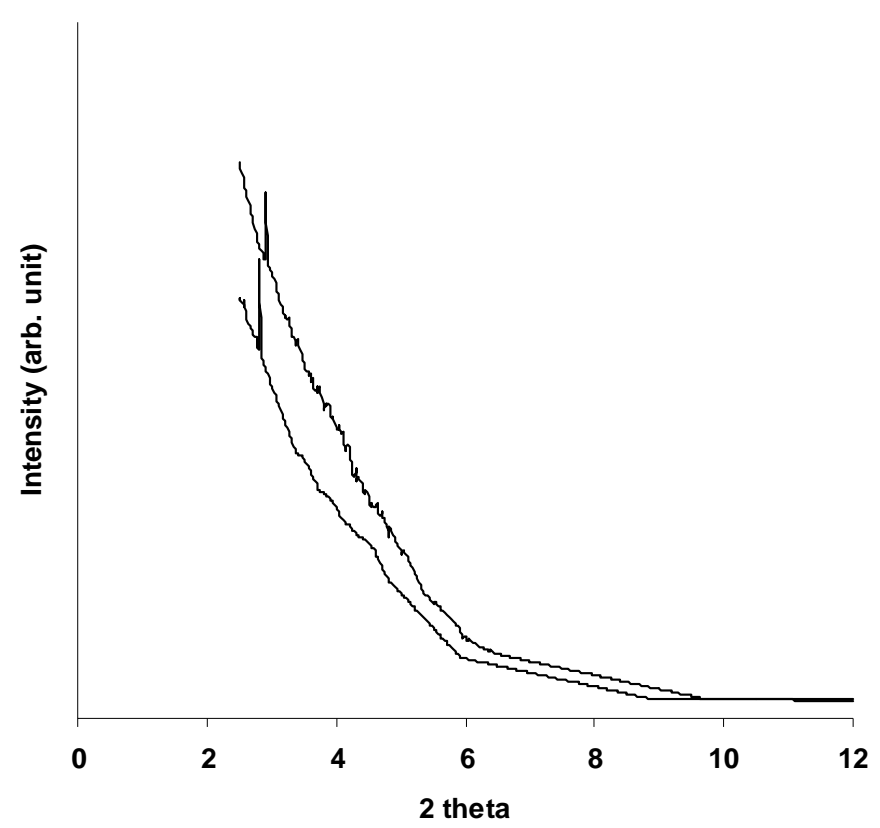

Figure 11. XRD of (a) MM-PP + $3 \mathrm{wt} \%$ and (b) HM-PP $+3 \mathrm{wt} \%$ clay processed at $245^{\circ} \mathrm{C}$.

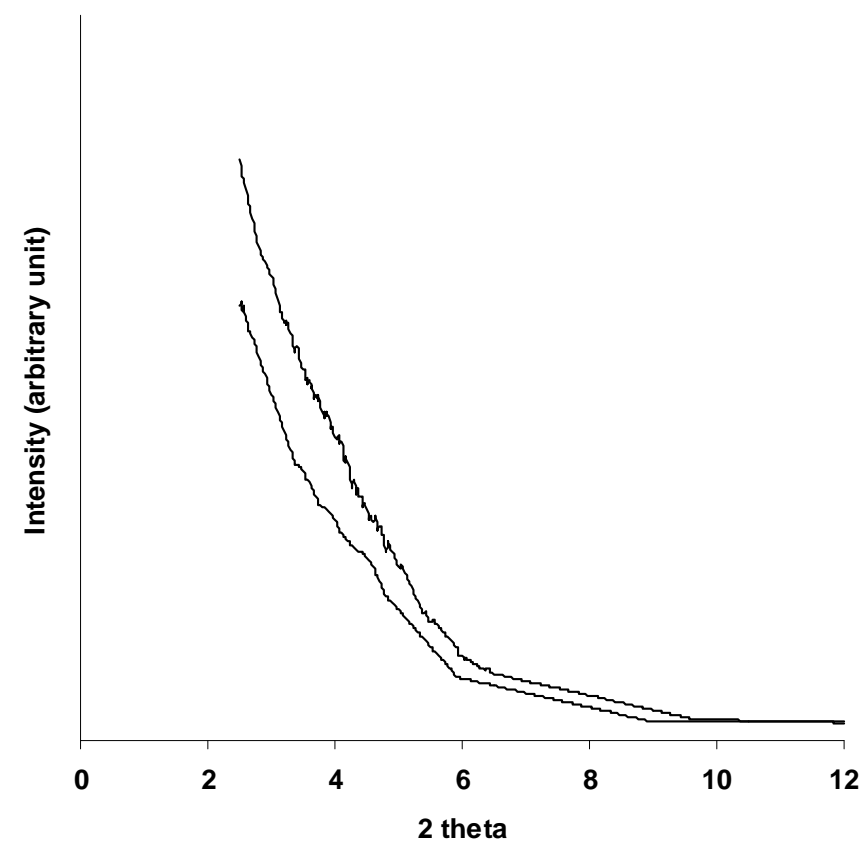

Figure 12. XRD of (a) MM-PP and (b) HM-PP with 3 wt \% clay processed at $120 \mathrm{rpm} / 230^{\circ} \mathrm{C}$.

temperature of composites shows the intercalated structure and whereas higher shear induced force composites shows the exfoliated structure. These pictures further support the XRD result. In high temperature processing, the separation of nanolayers can occurs only though the diffusion mechanisms, and the higher molecular weight polymer might face considerable difficulties in diffusing into the inter gallery regions of nanoclays and hence re- sulted in an intercalated structure. Whereas in high shear force induced nanocomposites processing, the increased shear force is sufficiently high enough to break the particles into smaller size, or to delaminate the clay nanolayers. Therefore high shear force can favour the formation of an exfoliated structure in high molecular weight polymer composites. The resultant tensile properties due to these processing effects is shown in Table 6 (Case 1 is 


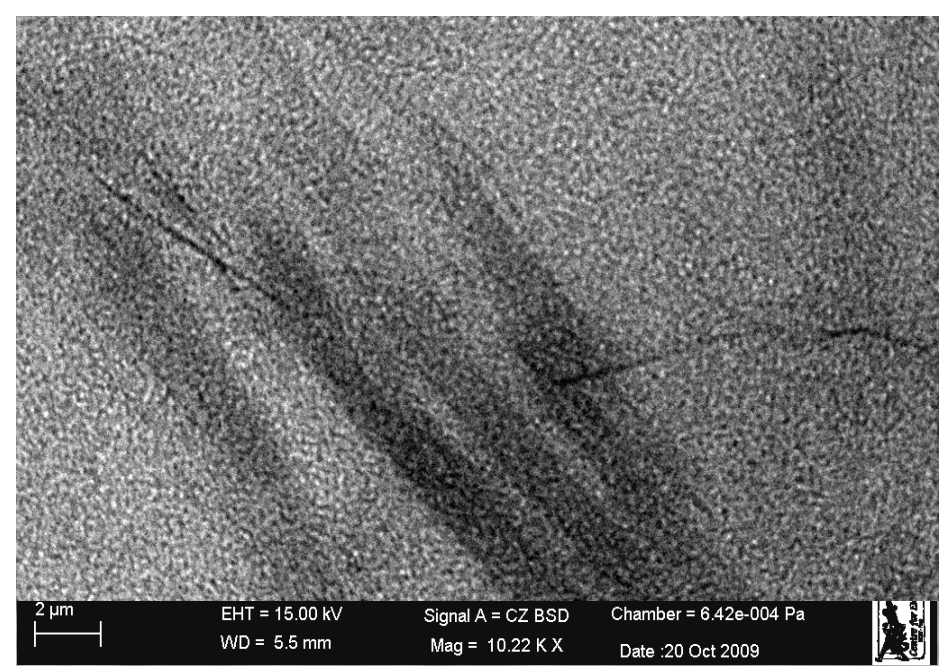

(a)

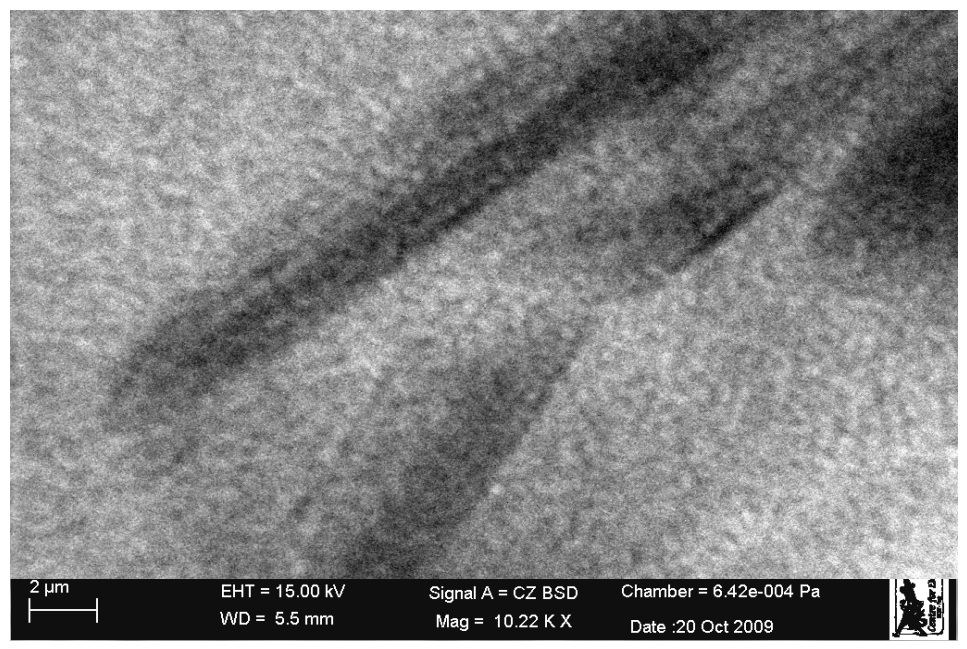

(b)

Figure 13. TEM of HM-PP + $3 \mathrm{wt} \%$ clay processed at (a) $245^{\circ} \mathrm{C} / 80 \mathrm{rpm}$ and (b) $230^{\circ} \mathrm{C} / 120 \mathrm{rpm}$.

Table 6. Effect of processing parameters on tensile properties.

\begin{tabular}{ccccccc}
\hline \multirow{2}{*}{ Material } & \multicolumn{2}{c}{ Modulus, GPa } & \multicolumn{2}{c}{ Strength, MPa } & \multicolumn{2}{c}{ Elongation, \% } \\
\cline { 2 - 7 } & Case 1 & Case 2 & Case 1 & Case 2 & Case 1 & Case 2 \\
\hline MM-PP + 1\% clay & 1.56 & 1.61 & 39 & 44 & 241 & 256 \\
MM-PP + 3\% clay & 1.90 & 1.97 & 41 & 47 & 261 & 270 \\
HM-PP + 1\% clay & 1.38 & 1.44 & 48 & 51 & 287 & 301 \\
HM-PP + 3\% clay & 1.82 & 1.93 & 49 & 56 & 293 & 306 \\
\hline
\end{tabular}

high processing temperature and Case 2 is high shear force). The result shows improved property enhancement of composites in high shear force condition due to the formation of an exfoliated nanocomposite structure.

\subsection{Effect of Synthetic Methods on Structure and Properties of Nanocomposites}

In a special case, the effect of synthetic methods on the 
properties is studied. Here, two synthetic methods are compared, namely extrusion process and casting process. Table 7 shows the effect of synthetic methods on tensile properties of composites. It shows that the extrusion method shows improved properties than that of casting method. The possible reason for low property in the casting method is examined by studying the morphology of the clay particles in PP matrix using TEM. Figure 14 shows the TEM of $3 \mathrm{wt} \%$ nanoclay dispersion in LM-PP matrix and shows the existence of intercalated nanocomposites structure with agglomerated clay particles. This agglomerated structure could have caused low property in the composites. This effect is caused due to the less shear force during mixing of clay particles and molten polymer.

\section{Conclusions}

A relatively new class of material consisting of polypropylene as matrix material and the nanoclay as reinforcement filler is prepared by using extrusion or casting methods. The result shows that the addition of nanoclays increases the melt viscosity of the all the type of PP samples. Nanoclay acts as nucleating agent in LM-PP composites due to the increase of $\%$ crystallization and rate of crystallinity of LM-PP samples. These nucleating effects of nanoclays in PP samples were studied using Avarami's equation. The kinetic study shows reduced nucleating effect of nanoclays in higher molecular weight PP samples. Exfoliated structure forms in LM-PP composite series, and where as proper improvement of shear force in extrusion proved exfoliated structure in higher molecular weight PP composite series. The low shear force and agglomeration of particles causes reduced tensile properties of melt cast samples over extruded composite.

The outcome of this work suggests that the nanoclays acts as a viable nanofiller in the development of particle filled polymer composites. The nanoclay distribution in matrix is depended on molecular weight of the polymer, processing or synthetic conditions. The nanoclays im-

Table 7. Effect of synthetic methods on tensile properties.

\begin{tabular}{|c|c|c|c|c|c|c|}
\hline \multirow{2}{*}{ Material } & \multicolumn{2}{|c|}{ Modulus, GPa } & \multicolumn{2}{|c|}{ Strength, MPa } & \multicolumn{2}{|c|}{ Elongation } \\
\hline & Extrusion & Casting & Extrusion & Casting & Extrusion & Casting \\
\hline LM-PP $+1 \%$ clay & 1.80 & 1.62 & 33 & 31 & 146 & 141 \\
\hline LM-PP $+3 \%$ clay & 2.30 & 2.18 & 36 & 33 & 163 & 156 \\
\hline MM-PP $+1 \%$ clay & 1.61 & 1.43 & 44 & 41 & 256 & 239 \\
\hline MM-PP $+3 \%$ clay & 1.97 & 1.72 & 47 & 45 & 270 & 256 \\
\hline $\mathrm{HM}-\mathrm{PP}+1 \%$ clay & 1.44 & 1.21 & 51 & 49 & 301 & 287 \\
\hline HM-PP $+3 \%$ clay & 1.93 & 1.73 & 56 & 51 & 306 & 293 \\
\hline
\end{tabular}

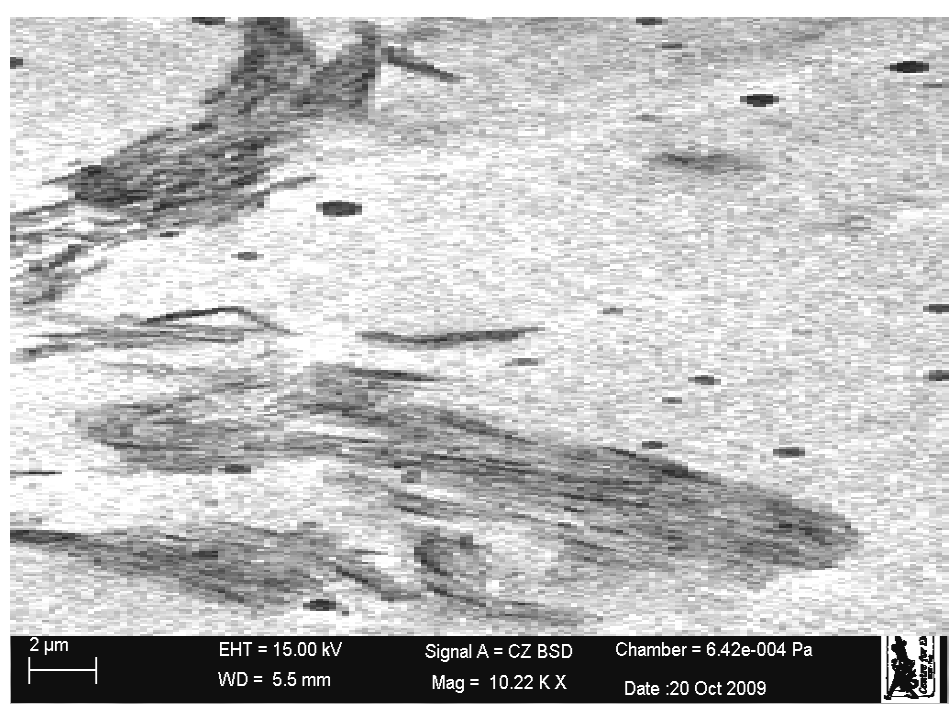

Figure 14. TEM of melt cast LM-PP + 3 wt \% nanoclay. 
proves the crystallization rate and there by useful for molding engineering components that require higher crystallization rate during processing.

\section{Acknowledgements}

Authors gratefully acknowledges the financial support provided by National Research Foundation-NRF (Grant No: 71599) of South Africa for carrying out this work.

\section{REFERENCES}

[1] E. Chabert, M. Bornert, E. Bourgeat-Lami, J. -Y. Cavaille, R. Dendievel, C. Gauthier, J. L. Putaux and A. Zaoui, "Filler-Filler Interactions and Viscoelastic Behavior of Polymer Nanocomposites," Materials Science and Engineering A, Vol. 381, No. 1-2, 15, September 2004, pp. 320-330.

[2] J. Jordan, I. Karl Jacob, R. Tannenbaum, M. A. Sharaf and I. Jasiuk, "Experimental Trends in Polymer Nanocomposites-A Review," Materials Science and Engineering A, Vol. 393, No. 1-2, 25 February 2005, pp. 1-11. doi:10.1016/j.msea.2004.09.044

[3] G. T. Wang, Hong-Yuan Liu, Nicolas Saintier and Yiu-Wing Mai, "Cyclic Fatigue of Polymer Nanocomposites," Engineering Failure Analysis, Vol. 16, No. 8, December 2009, pp. 2635-2645.

[4] R. Qiao and L. C. Brinson, "Simulation of Interphase Percolation and Gradients in Polymer Nanocomposites," Composites Science and Technology, Vol. 69, No. 3-4, March 2009, pp. 491-499.

doi:10.1016/j.compscitech.2008.11.022

[5] S. Pavlidou and C. D. Papaspyrides, "A Review on Polymer-Layered Silicate Nanocomposites," Progress in Polymer Science, Vol. 33, No. 12, December 2008, pp. 1119-1198. doi:10.1016/j.polymdegradstab.2005.04.040

[6] J. G. Zhang, D. D. Jiang and C. A. Wilkie, "Fire Properties of Styrenic Polymer-Clay Nanocomposites Based on an Oligomerically-Modified Clay," Polymer Degradation and Stability, Vol. 91, No. 2, February 2006, pp. 358-366. doi:10.1016/j.polymdegradstab.2005.04.040

[7] E. M. Araujo, R. Barbosa, A.W.B. Rodrigues, T. J. A. Melo and E. N. Ito, "Processing and Characterization of Polyethylene/Brazilian Clay Nanocomposites", Materials Science and Engineering: A, Vol. 445-446, 15 February 2007, pp. 141-147. doi:10.1016/j.msea.2006.09.012

[8] R. Sarathi, R. K. Sahu and P. Rajeshkumar, "Understanding the Thermal, Mechanical and Electrical Properties of Epoxy Nanocomposites," Materials Science and Engineering: A, Vol. 445-446, 15 February 2007, pp. 567-578. doi:10.1016/j.msea.2006.09.077

[9] S. -J. Park, B. -J. Kim, D. -I. Seo, K. -Y. Rhee and Y. -Y. Lyu, "Effects of a Silane Treatment on the Mechanical Interfacial Properties of Montmorillonite/Epoxy Nanocomposites", Materials Science and Engineering: A, Vol. 526, No. 1-2, 25 November 2009, pp. 74-78.

[10] Y. C. Hu, L. Shen, H. Yang, M. Wang, T. X. Liu, T. Li- ang and J. Zhang, "Nanoindentation Studies on Nylon 11/Clay Nanocomposites," Polymer Testing, Vol. 25, No. 4, June 2006, pp. 492-497. doi:10.1016/j.polymertesting.2006.02.005

[11] Y. H. Lee, T. Kuboki, C. B. Park, M. Sain and M. Kontopoulou, "The Effects of Clay Dispersion on the Mechanical, Physical, and Flame-Retarding Properties of Wood Fiber/Polyethylene/Clay Nanocomposites," Journal of Applied Polymer Science, Vol. 118, No. 1, 2010, pp. 452-461.

[12] L. B. de Paiva, A. R. Morales, R. Francisco and V. Díaz, "Organoclays: Properties, Preparation and Applications," Applied Clay Science, Vol. 42, No. 1-2, December 2008, pp. 8-24. doi:10.1016/j.clay.2008.02.006

[13] L. B. de Paiva, A. R. Morales and T. R. Guimaraes, "Structural and Optical Properties of Polypropylene-Montmorillonite Nanocomposites," Materials Science and Engineering: A, Vol. 447, No. 1-2, 25, February 2007, pp. 261-265.

[14] L. Raka, G. Bogoeva-Gaceva, K. Lu and J. Loos, "Characterization Of Latex-Based Isotactic Polypropylene/Clay Nanocomposites", Polymer, Vol. 50, No. 15, 17 July 2009, pp. 3739-3746. doi:10.1016/j.polymer.2009.05.044

[15] Y. Dong and D. Bhattacharyya, "Dual Role of Maleated Polypropylene in Processing and Material Characterisation of Polypropylene/Clay Nanocomposites," Materials Science and Engineering: A, Vol. 527, No. 6, 15 March 2010, pp. 1617-1622. doi:10.1016/j.msea.2009.10.043

[16] D. Garcia-Lopez, O. Picazo, J. C. Merino and J. M. Pastor, "Polypropylene-Clay Nanocomposites: Effect of Compatibilizing Agents on Clay Dispersion," European Polymer Journal, Vol. 39, No. 5, May 2003, pp. 945-950.

[17] J. Ma, E. Bilotti, T. Peijs and J. A. Darr, "Preparation of Polypropylene/Sepiolite Nanocomposites Using Supercritical $\mathrm{CO}_{2}$ Assisted Mixing," European Polymer Journal, Vol. 43, No. 12, December 2007, pp. 4931-4939. doi:10.1016/S0014-3057(02)00333-6

[18] C. O. Rohlmann, M. F. Horst, L. M. Quinzani and M. D. Failla, "Comparative Analysis of Nanocomposites Based on Polypropylene and Different Montmorillonites," European Polymer Journal, Vol. 44, No. 9, September 2008, pp. 2749-2760. doi:10.1016/j.eurpolymj.2008.07.006

[19] S. G. Lei, S. V. Hoa and M. -T. Ton-That, "Effect of Clay Types on the Processing and Properties of Polypropylene Nanocomposites," Composites Science and Technology, Vol. 66, No. 10, August 2006, pp. 1274-1279 doi:10.1016/j.compscitech.2005.09.012

[20] Y. X. Zhou, M. I. Jeelani and S. Jeelani, "Development of Photo Micro-Graph Method to Characterize Dispersion of Cnt in Epoxy," Materials Science and Engineering: A, Vol. 506, No. 1-2, 25, April 2009, pp. 39-44.

[21] L. Cauvin, D. Kondo, M. Brieu and N. Bhatnagar, "Mechanical Properties of Polypropylene Layered Silicate Nanocomposites: Characterization and Micro-Macro Modelling," Polymer Testing, Vol. 29, No. 2, April 2010, 
pp. 245-250. doi:10.1016/j.polymertesting.2009.11.007

[22] L. Cauvin, D. Kondo, M. Brieu and N. Bhatnagar, "Mec-Hanical Behavior of a PP Platelet-Reinforced Nanocomposite: Experimental Characterization and Two Scale Modeling of Linear and Non-Linear Response", Materials Science and Engineering: A, Vol. 527, No. 4-5, 15 February 2010, pp. 1102-1108. doi:10.1016/i.msea.2009.09.036

[23] L. L. Cui and D. R. Paul, "Evaluation of Amine Functionalized Polypropylenes as Compatibilizers for Polypropylene Nanocomposites," Polymer, Vol. 48, No. 6, 8 March 2007, pp. 1632-1640. doi:10.1016/j.polymer.2007.01.036

[24] M. Modesti, A. Lorenzetti, D. Bon and S. Besco, "Thermal Behaviour of Compatibilised Polypropylene Nanocomposite: Effect of Processing Conditions," Polymer
Degradation and Stability, Vol. 91, No. 4, April 2006, pp. 672-680. doi:10.1016/j.polymdegradstab.2005.05.018

[25] R. D. K. Misra, Q. Yuan, J. Chen and Y. Yang, "Hierarchical Structures and Phase Nucleation and Growth During Pressure-Induced Crystallization of Polypropylene Containing Dispersion of Nanoclay: The Impact on Physical and Mechanical Properties," Materials Science and Engineering: A, Vol. 527, No. 9, 15 April 2010, pp. 2163-2181.

[26] T. Labour, C. Gauthier, R. Seguela, G. Vigier, Y. Bomal and G. Orange, "Influence of the $\beta$ Crystalline Phase on the Mechanical Properties of Unfilled and $\mathrm{CACO}_{3}$-Filled Polypropylene. i. Structural and Mechanical Characterisation," Polymer, Vol. 42, No. 16, July 2001, pp. 71277135. doi:10.1016/S0032-3861(01)00089-1 